\title{
An Adaptive Interactive Multimedia System for Intelligent Environments
}

\author{
Dan-El Neil Vila Rosado, Margarita Esponda-Argüero, and Raúl Rojas
}

\begin{abstract}
We present a new interactive multimedia system for blended learning called PowerChalk. The goal of PowerChalk is to provide a robust, reliable, usable and sustainable multimedia technology to any intelligent environment focused on blended learning. The software architecture allows adapting the system to any hardware configuration and supports collaboration, communication, creativity and learning. In this sense, PowerChalk resolves many limitations of current e-learning systems, improves human-computer interaction for the management of different kinds of hardware and data and allows to implement different configurations for intelligent environments.
\end{abstract}

Index Terms-Multimedia technologies, blended learning, intelligent environments, pen-based user interfaces, modular programming.

\section{INTRODUCTION}

Technology has an increasing role in the evolution of education. Every new technological development opens new opportunities for lesson planning for teachers, and learning tools for students. Personal computers made computer aided learning possible, the Internet influenced the arise of e-learning, and the development of intelligent environments brings pedagogical advances. For such intelligent environments we need multimedia tools (classified as any combination of text, graphics, sound, animation and video delivered and controlled by the computer) that give high-performance, and high sensory attractiveness.

Learning theorists propose that to reach an objective, acquire a skill, or learn something new, the learner must be actively involved through practice in order to incorporate it into long term memory. The interaction while experimenting with the objective helps the learner to reach the objective and recall the information, skill or behavior that is supposed to be learned [1]. In this sense, the most interactive media tool is the chalkboard. In an abstract sense, a "blackboard or chalkboard" is an environment area where we can display some kind of data and ensure that the data and information stay available, thus providing context for any lecture or discussion. Therefore, the best tool to use in a intelligent environment is some kind of electronic chalkboard.

Because Intelligence Environments (IEs) are built using hardware and software to promote and improve the user experience, as well as facilitate participation and interaction among different kinds of users, we focus our efforts on

Manuscript received July 25, 2013; revised September 10, 2013.

The authors are with Freie Universität Berlin, Berlin, Germany (e-mail vila80@zedat.fu-berlin.de). support blended learning sessions: while still taking into account a "brick-and-mortar" learning system, computer-media activities are combined with face to face learning methods [2].

Following this motivation, we developed PowerChalk as an interactive media tool that provides an adaptive, modern, flexible, technology-friendly and pedagogical approach suitable to any intelligent environment; especially those focused on blended learning.

This paper is structured as follows. We review the related work in Section II. Then, in Section III we describe the PowerChalk software architecture, and in Section IV the different modules of the system. Finally, in Section V, the implications of our findings and further research are discussed.

\section{RELATED WORK}

Among applications which can provide a positive effect for learning in a intelligent environment focused on blended learning we find: educational tools, group decision making tools, graphical visualizations tools, etc. Currently there are few electronic systems and projects that offer a combination of collaboration platforms, interactive chalkboards and displays that enhance any discussion session.

Some representative examples of electronic systems and applications to give support to education and sharing information are:

NotePals. It is PhD. project developed by UCLA-Xerox [3] as an ink-based, lightweight note sharing system that gives group members easy access to each other's experiences through personal notes on digital assistants.

E-Cognocracy. Democratic system conceived for the purpose of extracting and diffusing the knowledge derived from a group of people. It was developed by Univ. Of Zaragoza [4].

K-Sketch. Interface design for creating informal animations from sketches. Developed by Univ. of Washington and Univ. Of California [5].

BumpTop. Virtual Desktop. Developed by University of Toronto [6].

PADDs. Digital documents that can be manipulated either on a computer screen, or on paper. University of Maryland [7].

SMART systems. Company of electronic whiteboards [8].

E-Chalk. Electronic chalkboard developed by the Freie Universität Berlin [9].

Cabri software. Interactive media software to create content faster to accompany any learning session with 
mathematical and physical objects or to provide activities as resources in 2 or 3 dimensions[10].

Sistema UNO. Education solutions. Grupo Santillana [11]

The systems above are specialized to a very specific task and have different limitations, among which we mention: cost, hardware or software limitations, inefficient software architecture, they can only work with certain types of data, lack proper software engineering, and present difficulty to evolve or update for developers or end-users.

PowerChalk transforms any learning session into a visual and reliable communication tool. It is a multi-platform software that can be configured to any screen system (computer, laptop, tablet-PC, multi-screen hardware, etc.). The software architecture allows distributed development, data efficient management and friendly update system.

\section{SofTWARE ARCHITECTURE}

PowerChalk has a modularized architecture for distributed development based in Java-NetBeans technology [12]. We solved the design problems of analyzed E-learning applications through design patterns like navigation, composition, semantic zooming, lookup, etc. [13]. The goals of PowerChalk are:

- Provide a robust, reliable, usable and sustainable system with an efficient software structure.

- Provide the platform with a set of tools that let the end-users analyze complex miscellaneous information quickly and insert relevant notes (Rich Client Platform).

- Provide the PowerChalk with a communication module to share information and have real-time collaborative sessions.

Considering these goals, we can attain a high-performance system for teaching and learning. A modular application like Power Chalk is composed of smaller, separated chunks of code that are well isolated. They can then be developed in separated teams with their own life cycle, and their own schedule. The results can then be assembled together by a separate entity [14].

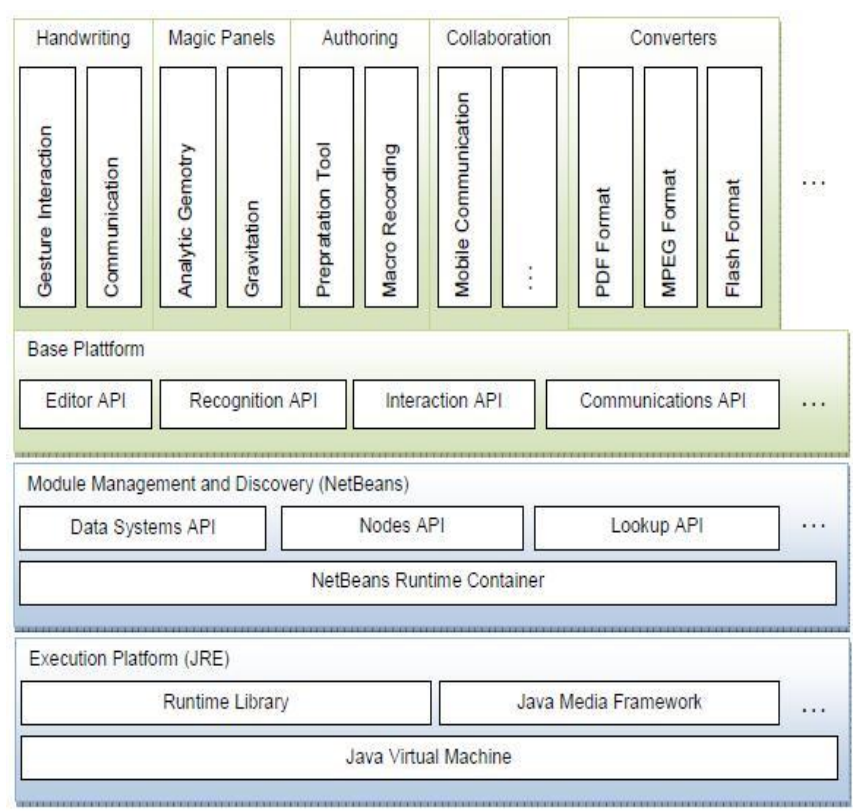

Fig. 1. PowerChalk software structure.
Such a modular architecture has the followings advantages:

- It simplifies the creation of new features.

- It makes it easy for users to add and remove features. With this characteristic the user can modify the different tools: the electronic ink, the PDF viewer, the image reader, and more.

- It makes it easy to update existing features.

With these benefits PowerChalk becomes a modern, configurable approach to any e-learning situation, lesson-planning or intelligent environment.

The architecture of PowerChalk is shown in Fig. 1.

\section{Modules}

In [15] an architecture has been proposed as a consequence of a methodology for the inclusion of Artificial Intelligence in Ambiente Intelligence environments. This architecture considers several modules. The first is related with the acquisition of data, information and even knowledge. A second module is related with the storage, conversion, and handling of the data-information-knowledge. The third one is involved with the necessary operations, and the last one is focused on actuation and interaction. The PowerChalk modules satisfy this architecture in order of support usability and end-user satisfaction.

In the PowerChalk system, the modules work together to improve our experience as end-user. The set of modules that allows to adapt PowerChalk to any hardware of an intelligent environment are the following:

\section{A. Main Editor}

The base of any intelligent environment for e-learning is an interactive multimedia area and electronic ink. We have developed an editor area for the PowerChalk canvas where we can add electronic ink components, several multimedia objects (images, keyboard input, etc.) and edit functionalities (Fig. 2). The editor includes pen-based applications to process the different kinds of objects through annotation, correction, condensation, organization, zoom abilities, print options (normal and PDF converter) and creation of slides.

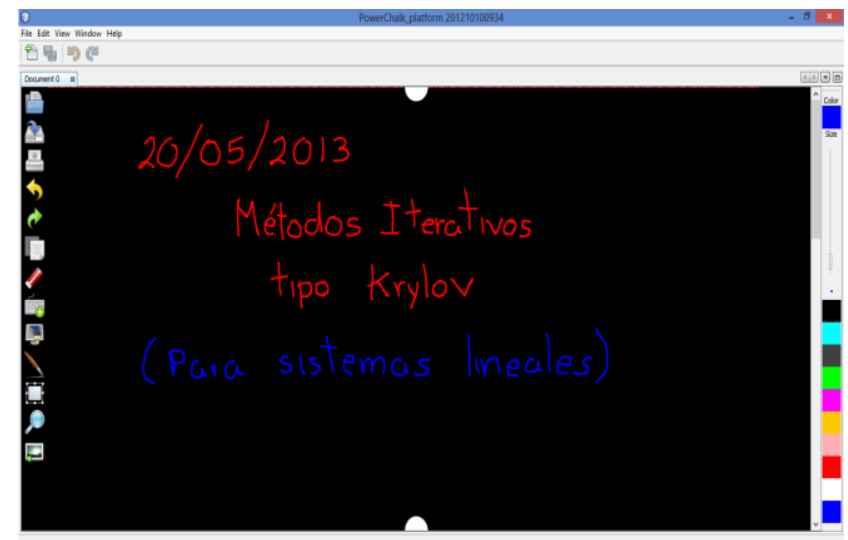

Fig. 2. A view of the main editor.

The main editor has a multiple overlapping layer mechanism to show different levels of multimedia data and allow visualizations, annotation, or underlining. Identification and processing of the different layers in the 
document structure of a PowerChalk session allows flexible data management over different kind of hardware like a data wall, laptop, tablet-PC, etc.

An intelligent agent for recognizing and adapt handwriting user interface prototypes was developed. This module works over the PowerChalk canvas to recognize paths delineated by users interactively. For the moment, we restrict the recognition to unistroke gestures that unfold over time. The recognition and interpretation of these gestures is editable and sustainable.

To endow PowerChalk with an informal animation tool we merge the structured 2D graphics framework Piccolo2D [16] with an efficient lookup system. The design of the object structure with the lookup system in PowerChalk let us to implement commands to animate objects over the PowerChalk-canvas on the fly, for example, we can animate affine transformations over an object and composite a set of elements. The result of the animation can be viewed in real time.

\section{B. Pen Tablets Module}

PowerChalk has a library for accessing pen/digitizer tablets and pointing devices using Java. Its key features are Event/Listener architecture and the fact that device access is implemented through providers in different operating systems (Linux, Windows, Wacom tablets, Hanvon tablets, etc.) and hardware devices (Fig. 3). Editor tools that interact with the pen tablet have also been developed; example of this is to open a color chooser through a click with pressure or a selection tool.

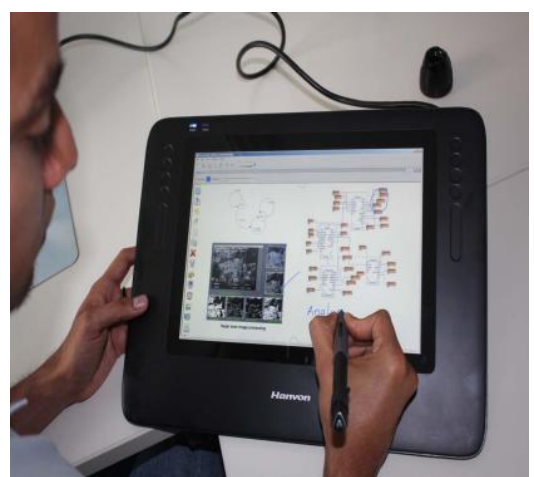

Fig. 3. PowerChalk in a digital tablet.

PowerChalk has been used in PC computers, tablet-PC systems and data walls for lectures at FU-Berlin by teachers and students in order to test the functionality and practicality.

\section{Multi-Screen Manager Module}

The increasing complex e-learning environment drives the requirement for the presentation and interactive control of and endless stream of data to get reliable information. The rapid adoption of digital devices leads to use several screens to perform the same activities. Every kind of screen has unique benefits; therefore, together they enhance the user experience. In general, the user wants relevant, consistent and connected information across screens to improve decision making in the classroom. This module allows to use PowerChalk in several end-users hardware configurations giving accessibility and productivity in any collaborative learning session or data conferencing .
Through PowerChalk we can define a PowerChalk editor (main or secondary) for every screen of the intelligent environment.

The principal experiments with the Power Chalk Editor were conducted in a Data-wall with four screens that was built in a classroom for testing (Fig. 4).

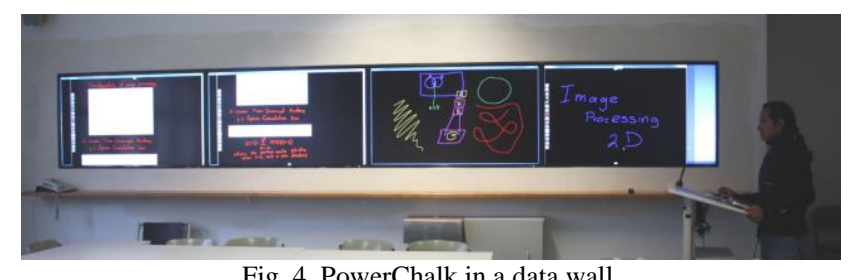

Fig. 4. PowerChalk in a data wall.

\section{Communication Module}

In a e-learning session the conversation and information on every screen of the intelligent environment are equally important. We want the user to be able to communicate and work together with richly formatted text, photos, videos, maps and more.

The structure of communication uses three channels: audio, video, and data in every PowerChalk canvas. The system has been tested transmitting audio and video over Real Time Transfer protocol (RTP), and the Java Media Framework API (JMF). The JMF enables audio, video and other time-based media to be added to any PowerChalk canvas. With this module in PowerChalk we can capture, playback, stream, share and transcode multiple media formats providing a powerful toolkit to develop scalable, cross-platform communication technology.

The hierarchy class of the objects or data (based on Piccolo 2D) let us to send any object through Internet and to show the same information in any other PowerChalk canvas [13]. This technology will allow us to transmit the PowerChalk-canvas data with high efficiency and synchronization with the other channels.

Otherwise, designing a web framework, web services and an update center for the PowerChalk platform will allow the users review, edit and build shared sets of recorded sessions. A recorded session could be a lecture, a discussion session over a set of data for group decision-making, an animation, or a set of data processed for gathering information. The communication module for developers is also responsible for supporting these activities.

PowerChalk has a "Wicket framework" (Apache Wicket) with proper mark-up/logic separation, a POJO data model, and a refreshing lack of XML. With this kind of application, the end-user is able to publish any work session to a general public in an easy way, for example: the classroom notes or homework.

Through the web services the user can integrate computer applications written in different languages and run on different platforms. Web services are independent from language and platform because vendors have agreed on common web service standards. PowerChalk works with RESTful Web Services and SOAP-based Web services.

Running the update center will check if there are new modules or new versions of already installed modules available. From new or updated modules, the user could 
select, download, and install them. We developed an update center module for the PowerChalk structure over the HTTP protocol.

\section{E. Collaboration Modules}

A collaboration module for applications is a bridge module between PowerChalk and software like MATLAB, OCTAVE, MATHEMATICA, Gnuplot, etc. With this kind of modules we can send instructions for plotting functions, expression evaluation, solving equations, running a script, etc. and see the output on the PowerChalk canvas (Fig. 5). We have designed an API for this kind of modules. The call to Gnuplot, or another application is made through a keyboard input or handwriting information in the PowerChalk canvas.

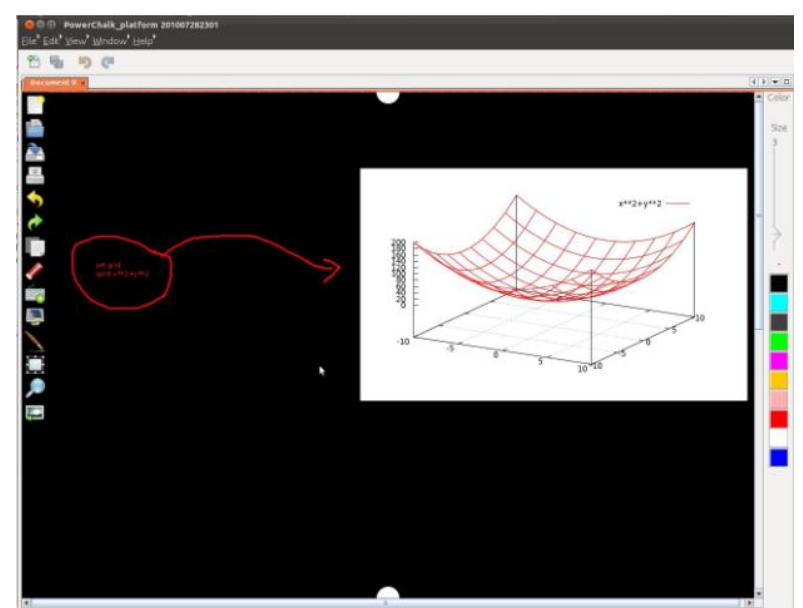

Fig. 5. Collaboration module example (PowerChalk-GNUPlot).

\section{F. Macros Module}

We use the term Macro to make available to the user, a sequence of quick notes (notes written in a prior time) or information (images, text, videos or PDF files) to use in a class session. The user interface of PowerChalk allows having the information for a class, available in an organized and efficient window system (Fig. 6).

It should be noted that we are able to add to the macro notes, or a page of notes written in a normal paper. This module can export a scanned file of the notes, binarize the image, make the segmentation of different annotations (if the annotations are split for a horizontal line) and make it available to the macro.

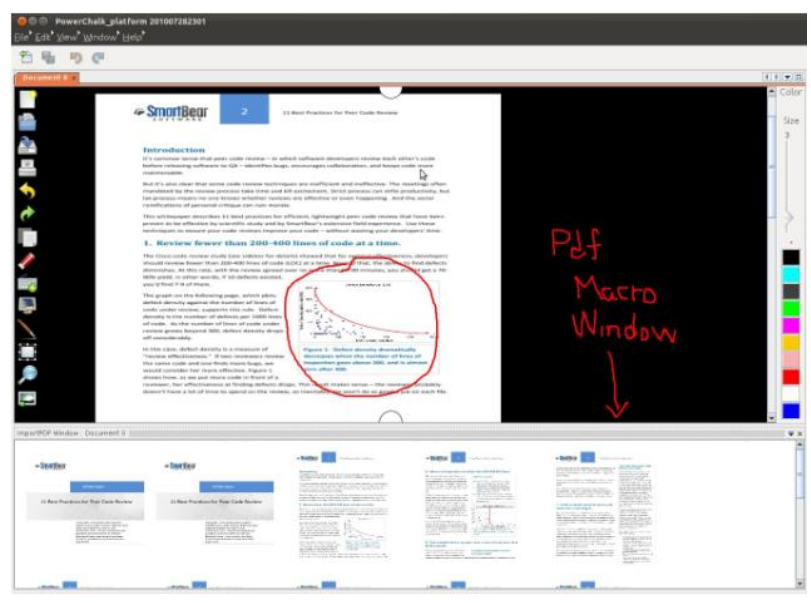

Fig. 6. Macros module.

\section{CONCLUSIONS AND FutURE WORK}

PowerChalk preserves the pedagogical benefits of the traditional chalkboard and provides the possibility to show not only results or isolated ideas but the train of thoughts. Combining the advantages of an interactive multimedia system and the faculty to be installed on different hardware allows it to be a useful and efficient system to be embedded into any intelligent environment, whether on a personal computer, or a complete working space.

Test in Windows, Linux and Mac systems were conducted, also with different hardware configurations (tablet-PC, data wall, notebooks), and end-users. The consensus is that PowerChalk it is a collaborative, robust, reliable, usable and sustainable interactive multimedia system for blended learning and also a friendly tool to review and share information on-line or off-line. It is a reliable base system for several intelligent environments.

It must be pointed out that the modular structure of PowerChalk let us amend quickly any problem in the system. A complete usability test it is in progress, in order to assess in a measurable way the advantages of PowerChalk,. Also, we are increasing the efficient of every module. For example we have:

New tools for animating objects on the canva. The objective it is to provide a complete platform for quick sketching to the end-user.

Bridge modules to communicate PowerChalk with softwares like MATHEMATICA, MATLAB, OCTAVE, etc. are under construction.

Module support to display a PowerChalk-canvas in iPAD or SMARTPhones.

Because of this advantage new modules are being developed. Among the future modules we can find: Voice recognizer module, image processing module, and a Java compile module, etc. The idea is to transform the PowerChalk system into a complete intelligent platform for sharing and processing general information.

\section{REFERENCES}

[1] D. Walter, C. Lou, and O. C. James, The Systematic Design of Instruction, 6th Ed., Allyn \& Bacon, pp. 1-12, ISBN: 0205412742, 2005.

[2] S. Valerie, "Three fears about blended learning," The Washington Post, 22 September 2012.

[3] R. C. Davis et al., in Proc. the SIGCHI conference on Human factors in computing systems, 1999.

[4] A. T. Lanuza et al., REDALYC: Magazine Computación y Sistemas, vol. 12, no. 2, December 2008, pp. 183-191.

[5] R. C. Davis et al., K-sketch: A "kinetic" Sketch Pad for Novice Animators, 2008.

[6] A. Agarawala and B. Ravin, "Keepin' it Real: Pushing the Desktop Metaphor with Physics," in Proc. CHI 2006 - the ACM Conference on Human Factors in Computing Systems, Piles and the Pen, pp. 1283-1292, 2006.

[7] F. Guimbretiere, Computer Human Interactions Letter, ACM, vol. 5, issue 2, 2003, pp. 51-60.

[8] Smarttech. [Online]. Available: http://smarttech.com/

[9] K. Jantz, G. Friedland, and R. Rojas, "Ubiquitous Pointing and Drawing," International Journal on Emerging Technologies for Learning (iJET), ISSN: 1863-0383, Wien, Austria 2007.

[10] Cabri. [Online]. Available: http://www.cabri.com

[11] Sistemauno. [Online]. Available: http://www.sistemauno.com/

[12] Netbeans. [Online]. Available: www.netbeans.org

[13] Gamma et al., Design patterns: Elements of reusable object oriented software, Addison-Wesley professional computer series, 1994. 
[14] T. Boudreau, J. Tulach, and G. Wielenga, "Rich Client Programming," Plugging into the NetBeans Platform, Prentice Hall 2006, ISBN: $0-13-235480$

[15] C. Ramos "An Architecture for Ambient Intelligent Environments," Advances in Soft Computing, vol. 51, 2009, pp. 30-38, ISBN: 978-3-540-85866-9

[16] B. Bederson, J. Grosjean, and J. Meyer, "Toolkit Design for Interactive Structured Graphics," IEEE Transactions on Software Engineering, 2004.

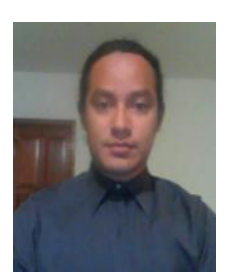

Dan-EI Neil Vila Rosado was born in Campeche, Campeche, Mexico in 1980. He is a PhD student from Freie Universität Berlin, localized in Berlin, Germany since 2008. He has a bachelor in Mathematics from University of Guanajuato (1998-2003) and a Maste degree in Computer Sciences and Industrial Mathematics by the Research Center in Mathemathics (Guanajuato, Mexico 2004-2006). The author's major fields of study are: Optimization, Soft computing, Artificial Intelligence and Educational Technology.

He was couch in the Regional Mathematics Olympiad Campeche (1998 -2000), coworker in the Center for Research in Mathematics A.C. Department of Technology Services (2000-2004). Also, he was software developer in the Consulting and Tecnology group (CONTECK 2005-2007) and project coordinator (2007-2008). He worked like teacher, research asistant and head of division in the University of Guanajuato (2002-2007) and Institute Technology of Irapuato (2007-2008).

Ms.C. Vila has published refereed conference papers supporting his work on robotics, optimization artificial intelligence and e-learning.

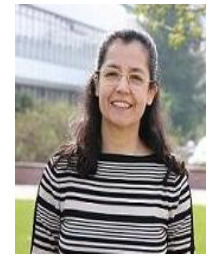

Margarita Esponda-Argüero is a professor of algorithms and programming languagues at Freie Universität Berlin since 2009. Previously, she held professorships at Technical University of Gießen-Friedberg and at the Technical University of Wildau.

Margarita Esponda obtained his bachelor degree in Mathematics and Physics from the National Polytechnic Institute of Mexico and the master degree in Informatics from the Technical University of Berlin. She received a PhD in Informatics from FU Berlin in 2004.

Prof. Esponda has been working on thechniques for visualizin data structures in algorithmic animation systems, electronic voting systems and multimedia technologies. She has published refereed conference and journal papers supporting his work on educational computing research, history of computing and information visualization.

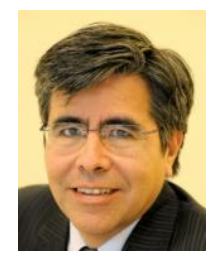

Raúl Rojas is a professor of Artificial Intelligence at Freie Universität Berlin since 1997. He received a $\mathrm{PhD}$ in Economics from FU Berlin in 1989, where he also obtained his Habilitation degree in Computer Science in 1994.

Prof. Rojas has been active in the field of Artificial Intelligence since 1986, having been a member of the Prolog Machine team at GMD-First (now Fraunhofer Gesellschaft). He has published extensively about neural networks, robotics, intelligent systems and the history of computing.

Rojas is a Member of the Mexican Academy of Sciences. He received the Technology Transfer Prize 2008 from the Berlin Technology Foundation and the Gold Medal Heberto Castillo from Mexico City for his contributions to robotics. 